\title{
BOUNDED ENGEL ELEMENTS IN RESIDUALLY FINITE GROUPS
}

\author{
RAIMUNDO BASTOS AND DANILO SILVEIRA
}

\begin{abstract}
Let $q$ be a prime. Let $G$ be a residually finite group satisfying an identity. Suppose that for every $x \in G$ there exists a $q$-power $m=m(x)$ such that the element $x^{m}$ is a bounded Engel element. We prove that $G$ is locally virtually nilpotent. Further, let $d, n$ be positive integers and $w$ a non-commutator word. Assume that $G$ is a $d$-generator residually finite group in which all $w$-values are $n$-Engel. We show that the verbal subgroup $w(G)$ has $\{d, n, w\}$ bounded nilpotency class.
\end{abstract}

\section{INTRODUCTION}

Given a group $G$, an element $g \in G$ is called a (left) Engel element if for any $x \in G$ there exists a positive integer $n=n(x, g)$ such that $[x, n g]=1$, where the commutator $[x, n g]$ is defined inductively by the rules

$$
\left[x,_{1} g\right]=[x, g]=x^{-1} g^{-1} x g \quad \text { and, for } n \geq 2, \quad\left[x,_{n} g\right]=\left[\left[x,_{n-1} g\right], g\right] .
$$

If $n$ can be chosen independently of $x$, then $g$ is called a (left) $n$-Engel element, or more generally a bounded (left) Engel element. The group $G$ is an Engel group (resp. an $n$-Engel group) if all its elements are Engel (resp. $n$-Engel).

A celebrated result due to Zelmanov [24, 25, 26] refers to the positive solution of the Restricted Burnside Problem (RBP for short): every residually finite group of bounded exponent is locally finite. The group $G$ is said to have a certain property locally if any finitely generated subgroup of $G$ possesses that property. An interesting result in this context, due to Wilson [21], states that every $n$-Engel residually finite group is locally nilpotent. Another result that was deduced following the positive solution of the RBP is that given positive integers $m, n$, if $G$ is a residually finite group in which for every $x \in G$ there exists a positive integer $q=q(x) \leqslant m$ such that $x^{q}$ is $n$-Engel, then $G$

2010 Mathematics Subject Classification. 20F45, 20E26.

Key words and phrases. Engel elements, Residually finite groups.

The first author was partially supported by FAPDF/Brazil. 
is locally virtually nilpotent [1]. We recall that a group possesses a certain property virtually if it has a subgroup of finite index with that property. For more details concerning Engel elements in residually finite groups see [1, 2, 3, 17, 18].

One of the goals of the present article is to study residually finite groups in which some powers are bounded Engel elements. We establish the following result.

Theorem A. Let $q$ be a prime. Let $G$ be a residually finite group satisfying an identity. Suppose that for every $x \in G$ there exists a $q$ power $m=m(x)$ such that the element $x^{m}$ is a bounded Engel element. Then $G$ is locally virtually nilpotent.

A natural question arising in the context of the above theorem is whether the theorem remains valid with $m$ allowed to be an arbitrary natural number rather than $q$-power. This is related to the conjecture that if $G$ is a residually finite periodic group satisfying an identity, then the group $G$ is locally finite (Zelmanov, [23, p. 400]). Note that the hypothesis that $G$ satisfies an identity is really needed. For instance, it is well known that there are residually finite $p$-groups that are not locally finite (Golod, [5]). In particular, these groups cannot be locally virtually nilpotent. Similar examples have been obtained independently by Grigorchuk, Gupta-Sidki and Sushchansky and are published in [6, 7, 20], respectively.

Recall that a group-word $w=w\left(x_{1}, \ldots, x_{s}\right)$ is a nontrivial element of the free group $F=F\left(x_{1}, \ldots, x_{s}\right)$ on free generators $x_{1}, \ldots, x_{s}$. A word is a commutator word if it belongs to the commutator subgroup $F^{\prime}$. A non-commutator word $u$ is a group-word such that the sum of the exponents of some variable involved in it is non-zero. A group-word $w$ can be viewed as a function defined in any group $G$. The subgroup of $G$ generated by the $w$-values is called the verbal subgroup of $G$ corresponding to the word $w$. It is usually denoted by $w(G)$. However, if $k$ is a positive integer and $w=x_{1}^{k}$, it is customary to write $G^{k}$ rather than $w(G)$.

There is a well-known quantitative version of Wilson's theorem, that is, if $G$ is a $d$-generator residually finite $n$-Engel group, then $G$ has $\{d, n\}$-bounded nilpotency class. As usual, the expression " $\{a, b, \ldots\}$ bounded" means "bounded from above by some function which depends only on parameters $a, b, \ldots$ ". We establish the following related result.

Theorem B. Let $d, n$ be positive integers and $w$ a non-commutator word. Assume that $G$ is a d-generator residually finite group in which all $w$-values are $n$-Engel. Then the verbal subgroup $w(G)$ has $\{d, n, w\}$ bounded nilpotency class. 
A non-quantitative version of the above theorem already exists in the literature. It was obtained in [3, Theorem C].

The paper is organized as follows. In the next section we describe some important ingredients of what are often called "Lie methods in group theory". Theorems A and B are proved in Sections 3 and 4, respectively. The proofs of the main results rely of Zelmanov's techniques that led to the solution of the RBP [24, 25, 26], Lazard's criterion for a pro- $p$ group to be $p$-adic analytic [9], and a result of Nikolov and Segal [13] on verbal width in groups.

\section{Associated Lie algebras}

Let $L$ be a Lie algebra over a field $\mathbb{K}$. We use the left normed notation: thus if $l_{1}, l_{2}, \ldots, l_{n}$ are elements of $L$, then

$$
\left[l_{1}, l_{2}, \ldots, l_{n}\right]=\left[\ldots\left[\left[l_{1}, l_{2}\right], l_{3}\right], \ldots, l_{n}\right] .
$$

We recall that an element $a \in L$ is called ad-nilpotent if there exists a positive integer $n$ such that $\left[x,{ }_{n} a\right]=0$ for all $x \in L$. When $n$ is the least integer with the above property then we say that $a$ is ad-nilpotent of index $n$.

Let $X \subseteq L$ be any subset of $L$. By a commutator of elements in $X$, we mean any element of $L$ that can be obtained from elements of $X$ by means of repeated operation of commutation with an arbitrary system of brackets including the elements of $X$. Denote by $F$ the free Lie algebra over $\mathbb{K}$ on countably many free generators $x_{1}, x_{2}, \ldots$ Let $f=f\left(x_{1}, x_{2}, \ldots, x_{n}\right)$ be a non-zero element of $F$. The algebra $L$ is said to satisfy the identity $f \equiv 0$ if $f\left(l_{1}, l_{2}, \ldots, l_{n}\right)=0$ for any $l_{1}, l_{2}, \ldots, l_{n} \in L$. In this case we say that $L$ is PI. Now, we recall an important theorem of Zelmanov [23, Theorem 3] that has many applications in group theory.

THEOREM 2.1. Let $L$ be a Lie algebra over a field generated by a finite set. Assume that $L$ is PI and that each commutator in the generators is ad-nilpotent. Then $L$ is nilpotent.

2.1. On Lie Algebras Associated with Groups. Let $G$ be a group and $p$ a prime. Let us denote by $D_{i}=D_{i}(G)$ the $i$-th dimension subgroup of $G$ in characteristic $p$. These subgroups form a central series of $G$ known as the Zassenhaus-Jennings-Lazard series (see [8, p. 250] for more details). Set $L(G)=\bigoplus D_{i} / D_{i+1}$. Then $L(G)$ can naturally be viewed as a Lie algebra over the field $\mathbb{F}_{p}$ with $p$ elements.

The subalgebra of $L(G)$ generated by $D_{1} / D_{2}$ will be denoted by $L_{p}(G)$. The nilpotency of $L_{p}(G)$ has strong influence in the structure of a finitely generated group $G$. According to Lazard [10] the nilpotency 
of $L_{p}(G)$ is equivalent to $G$ being $p$-adic analytic (for details see [10, A.1 in Appendice and Sections 3.1 and 3.4 in Ch. III] or [4, 1.(k) and 1.(o) in Interlude A]).

THEOREM 2.2. Let $G$ be a finitely generated pro-p group. If $L_{p}(G)$ is nilpotent, then $G$ is p-adic analytic.

Let $x \in G$ and let $i=i(x)$ be the largest positive integer such that $x \in D_{i}$ (here, $D_{i}$ is a term of the $p$-dimensional central series to $G$ ). We denote by $\tilde{x}$ the element $x D_{i+1} \in L(G)$. We now quote two results providing sufficient conditions for $\tilde{x}$ to be ad-nilpotent. The first lemma was established in [9, p. 131].

Lemma 2.3. For any $x \in G$ we have $(\operatorname{ad} \tilde{x})^{p}=\operatorname{ad}\left(\widetilde{x^{p}}\right)$. Consequently, if $x$ is of finite order $t$ then $\tilde{x}$ is ad-nilpotent of index at most $t$.

Corollary 2.4. Let $x$ be an element of a group $G$ for which there exists a positive integer $m$ such that $x^{m}$ is $n$-Engel. Then $\tilde{x}$ is adnilpotent.

The following result was established by Wilson and Zelmanov in [22].

LEMma 2.5. Let $G$ be a group satisfying an identity. Then for each prime number $p$ the Lie algebra $L_{p}(G)$ is $P I$.

\section{Proof of Theorem A}

Recall that a group is locally graded if every nontrivial finitely generated subgroup has a proper subgroup of finite index. Interesting classes of groups (e.g., locally finite groups, locally nilpotent groups, residually finite groups) are locally graded (see [11, 12] for more details).

It is easy to see that a quotient of a locally graded group need not be locally graded (see for instance [14, 6.19]). However, the next result gives a sufficient condition for a quotient to be locally graded [11].

Lemma 3.1. Let $G$ be a locally graded group and $N$ a normal locally nilpotent subgroup of $G$. Then $G / N$ is locally graded.

In [23], Zelmanov has shown that if $G$ is a residually finite $p$-group which satisfies a nontrivial identity, then $G$ is locally finite. Next, we extend this result to the class of locally graded groups.

LEMMA 3.2. Let $p$ be a prime. Let $G$ be a locally graded p-group which satisfies an identity. Then $G$ is locally finite.

Proof. Choose arbitrarily a finitely generated subgroup $H$ of $G$. Let $R$ be the finite residual of $H$, i.e., the intersection of all subgroups of finite index in $H$. If $R=1$, then $H$ is a finitely generated residually finite 
group. By Zelmanov's result [23, Theorem 4], $H$ is finite. So it suffices to show that $H$ is residually finite. We argue by contradiction and suppose that $R \neq 1$. By the above argument, $H / R$ is finite and thus $R$ is finitely generated. As $R$ is locally graded we have that $R$ contains a proper subgroup of finite index in $H$, which gives a contradiction. Since $H$ be chosen arbitrarily, we now conclude that $G$ is locally finite, as well. The proof is complete.

We denote by $\mathcal{N}$ the class of all finite nilpotent groups. The following result is a straightforward corollary of [21, Lemma 2.1] (see [15, Lemma 3.5] for details).

Lemma 3.3. Let $G$ be a finitely generated residually- $\mathcal{N}$ group. For each prime $p$, let $R_{p}$ be the intersection of all normal subgroups of $G$ of finite p-power index. If $G / R_{p}$ is nilpotent for each prime $p$, then $G$ is nilpotent.

We are now in a position to prove Theorem A.

Proof of Theorem A. Recall that $G$ is a residually finite group satisfying an identity in which for every $x \in G$ there exists a $q$-power $m=m(x)$ such that the element $x^{m}$ is a bounded Engel element. We need to prove that every finitely generated subgroup of $G$ is virtually nilpotent

Firstly, we prove that all bounded Engel elements (in $G$ ) are contained in the Hirsch-Plotkin radical of $G$. Let $H$ be a subgroup generated by finitely many bounded Engel elements in $G$, say $H=\left\langle h_{1}, \ldots, h_{t}\right\rangle$, where $h_{i}$ is a bounded Engel element in $G$ for every $i=1, \ldots, t$. Since finite groups generated by Engel elements are nilpotent [14, 12.3.7], we can conclude that $H$ is residually- $\mathcal{N}$. As a consequence of Lemma 3.3, we can assume that $G$ is residually-(finite $p$-group) for some prime $p$. Let $L=L_{p}(H)$ be the Lie algebra associated with the ZassenhausJennings-Lazard series

$$
H=D_{1} \geq D_{2} \geq \cdots
$$

of $H$. Then $L$ is generated by $\tilde{h}_{i}=h_{i} D_{2}, i=1,2, \ldots, t$. Let $\tilde{h}$ be any Lie-commutator in $\tilde{h}_{i}$ and $h$ be the group-commutator in $h_{i}$ having the same system of brackets as $\tilde{h}$. Since for any group commutator $h$ in $h_{1} \ldots, h_{t}$ there is a $q$-power $m=m(h)$ and a positive integer $n=n(h)$ such that $h^{m}$ is $n$-Engel, Corollary 2.4 shows that any Lie commutator in $\tilde{h}_{1} \ldots, \tilde{h}_{t}$ is ad-nilpotent. On the other hand, $H$ satisfies an identity and therefore, by Lemma 2.5, $L$ satisfies some non-trivial polynomial identity. According to Theorem $2.1 \mathrm{~L}$ is nilpotent. Let $\hat{H}$ denote the pro- $p$ completion of $H$. Then $L_{p}(\hat{H})=L$ is nilpotent 
and $\hat{H}$ is a $p$-adic analytic group by Theorem 2.2, By 4, 1.(n) and 1.(o) in Interlude A]), $\hat{H}$ is linear, and so therefore is $H$. Clearly $H$ cannot have a free subgroup of rank 2 and so, by Tits' Alternative [19], $H$ is virtually soluble. By [14, 12.3.7], $H$ is soluble. Since $h_{1}, \ldots, h_{t}$ have been chosen arbitrarily, we now conclude that all bounded Engel elements are in the Hirsch-Plotkin radical of $G$.

Let $H$ be a finitely generated subgroup of $G$, and $K$ be the subgroup generated by all bounded Engel elements (in $G$ ) contained in $H$. Now, we need to prove that $K$ is a nilpotent subgroup of finite index in $H$. By the previous paragraph, $K$ is locally nilpotent. By Lemma 3.1, $H / K$ is a locally graded $q$-group. Since $G$ satisfies a nontrivial identity, by Lemma 3.2, $H / K$ is finite and so, $K$ is finitely generated. From this we deduce that $K$ is nilpotent. The proof is complete.

\section{Proof of Theorem B}

Combining the positive solution of the RBP with the result 3 , Theorem $\mathrm{C}$ ] one can show that if $u$ is a non-commutator word and $G$ is a finitely generated residually finite group in which all $u$-values are $n$-Engel, then the verbal subgroup $u(G)$ is nilpotent. This section is devoted to obtain a quantitative version of the aforementioned result.

The proof of Theorem B require the following lemmas.

LEMMA 4.1. Let $d, m, n$ positive integers. Let $G$ be a d-generator residually finite group in which $x^{m}$ is $n$-Engel for every $x \in G$. Then the subgroup $G^{m}$ has $\{d, m, n\}$-bounded nilpotency class.

Proof. Let $H=G^{m}$. By [3, Theorem C], $H$ is locally nilpotent. Moreover, Lemma 3.1 ensures us that the quotient group $G / H$ is locally graded. By Zelmanov's solution of the RBP, locally graded groups of finite exponent are locally finite (see for example [12, Theorem 1]), and so $G / H$ is finite of $\{d, m\}$-bounded order. We can deduce from [14, Theorem 6.1.8(ii)] that $H$ has $\{d, m\}$-boundedly many generators. In particular, $H$ is nilpotent. In order to complete the proof, we need to show that $H$ has $\{d, m, n\}$-bounded class yet.

Note that there exists a family of normal and finite index subgroups $\left\{N_{i}\right\}_{i \in \mathcal{I}}$ in $G$ which are all contained in $H$ such that $H$ is isomorphic to a subgroup of the Cartesian product of the finite quotients $H / N_{i}$. We show that all quotients have $\{d, m, n\}$-bounded class. Indeed, we have $H / N_{i}=\left(G / N_{i}\right)^{m}$. Note that $H$ is $\{d, m\}$-boundedly generated. Thus, by [13, Theorem 1], $H / N_{i}$ is $\{d, m\}$-boundedly generated where any generator is an $m$-th power which is an $n$-Engel element. By [17, Lemma 2.2], there exists a number $c$ depending only on $\{d, m, n\}$ such 
that each factor $H / N_{i}$ has nilpotency class at most $c$. So $H$ is of nipotency class at most $c$, as well. The proof is complete.

A well known theorem of Gruenberg says that a soluble group generated by finitely many Engel elements is nilpotent (see [14, 12.3.3]). We will require a quantitative version of this theorem whose proof can be found in [16, Lemma 4.1].

LEMma 4.2. Let $G$ be a group generated by $m$ elements which are $n$ Engel and suppose that $G$ is soluble with derived length d. Then $G$ is nilpotent of $\{d, m, n\}$-bounded class.

For the reader's convenience we restate Theorem B.

Theorem B. Let $d, n$ be positive integers and $w$ a non-commutator word. Assume that $G$ is a d-generator residually finite group in which all $w$-values are $n$-Engel. Then the verbal subgroup $w(G)$ has $\{d, n, w\}$ bounded nilpotency class.

Proof. Let $w=w\left(x_{1}, \ldots, x_{r}\right)$ be a non-commutator word. We may assume that the sum of the exponents of $x_{1}$ is $k \neq 0$. Substitute 1 for $x_{2}, \ldots, x_{r}$ and an arbitrary element $g \in G$ for $x_{1}$. We see that $g^{k}$ is a $w$-value for every $g \in G$. Thus every $k$-th power is $n$-Engel in $G$. Lemma 4.1 ensures that $G^{k}$ has $\{d, n, w$,$\} -bounded nilpotency class.$

Following an argument similar to that used in the proof of Lemma 4.1 we can deduce that the verbal subgroup $w(G)$ is nilpotent. By Zelmanov's solution of the RBP, locally graded groups of finite exponent are locally finite (see for example [12, Theorem 1]), and so $G / G^{k}$ is finite of $\{d, w\}$-bounded order. Thus, the verbal subgroup $w(G)$ has $\{d, m, w\}$-bounded derived length.

Note that there exists a family of normal and finite index subgroups $\left\{N_{i}\right\}_{i \in \mathcal{I}}$ in $G$ that are all contained in $w(G)$ such that $w(G)$ is isomorphic to a subgroup of the Cartesian product of the finite quotients $w(G) / N_{i}$. We show that all quotients $w(G) / N_{i}$ have $\{d, n, w\}$ bounded class. Indeed, we have $w(G) / N_{i}=w\left(G / N_{i}\right)$. We also have $w(G)$ is $\{d, w\}$-boundedly generated. By [13, Theorem 3] each quotient $w(G) / N_{i}$ is $\{d, w\}$-boundedly generated by $w$-values which are $n$-Engel elements. Since $w(G)$ has $\{d, m, w\}$-bounded derived length, according to Lemma 4.2 we can deduce that $w(G) / N_{i}$ has $\{d, n, w\}$-bounded nilpotency class Thus, $w(G)$ has $\{d, n, w\}$-bounded nilpotency class, as well. This completes the proof.

\section{REFERENCES}

[1] R. Bastos, On residually finite groups with Engel-like conditions, Comm. Algebra, 44 (2016) 4177-4184. 
[2] R. Bastos, N. Mansuroğlu, A. Tortora, M. Tota, Bounded Engel elements in groups satisfying an identity, Arc. Math., 110 (2018) 311-318.

[3] R. Bastos, P. Shumyatsky, A. Tortora, M. Tota, On groups admitting a word whose values are Engel, Int. J. Algebra Comput., 23 (2013) 81-89.

[4] J. D. Dixon, M. P. F. du Sautoy, A. Mann, D. Segal, Analytic Pro-p Groups, Cambridge University Press, Cambridge, (1991).

[5] E.S. Golod, On nil-algebras and finitely approximable p-groups, Izv. Akad. Nauk SSSR Ser. Mat., 28 (1964) 273-276.

[6] R. I. Grigorchuk, On Burnside's problem on periodic groups, Functional Anal. Appl., 14 (1980) 41-43.

[7] N. Gupta, S. Sidki, On the Burnside problem for periodic groups, Math. Z., 182 (1983) 385-386.

[8] B. Huppert, N. Blackburn, Finite Groups II, Springer-Verlag, Berlin, (1982).

[9] M. Lazard, Sur les groupes nilpotents et les anneaux de Lie, Ann. Sci. École Norm. Sup., 71 (1954) 101-190.

[10] M. Lazard, Groupes analytiques p-adiques, IHES Publ. Math., 26 (1965) 389603.

[11] P. Longobardi, M. Maj and H. Smith, A note on locally graded groups, Rend. Sem. Mat. Univ. Padova, 94 (1995) 275-277.

[12] O. Macedońska, On difficult problems and locally graded groups, J. Math. Sci. (N.Y.), 142 (2007) 1949-1953.

[13] N. Nikolov, D. Segal, Powers in finite groups, Groups Geom. Dyn., 5 (2011) 501-507.

[14] D. J.S. Robinson, A course in the theory of groups, 2nd edition, SpringerVerlag, New York, (1996).

[15] P. Shumyatsky, Applications of Lie ring methods to group theory, in Nonassociative Algebra and Its Applications, (Eds R. Costa et al.), Marcel Dekker, New York, (2000) 373-395.

[16] P. Shumyatsky, D. S. Silveira, On finite groups with automorphisms whose fixed points are Engel, Arch. Math., 106 (2016) 209-218.

[17] P. Shumyatsky, A. Tortora, M. Tota, On varieties of groups satisfying an Engel type identity, J. Algebra, (2016), 447 (2016) 479-489.

[18] P. Shumyatsky, A. Tortora, M. Tota, Engel groups with an identity, Int. J. Algebra Comput., (2018), preprint avaible at arXiv:1805.12411 [math.GR].

[19] J. Tits, Free subgroups in linear groups, J. Algebra, 20 (1972) 250-270.

[20] V.I. Sushchansky, Periodic p-elements of permutations and the general Burnside problem, Dokl. Akad. Nauk SSSR, 247 (1979) 447-461.

[21] J.S. Wilson, Two-generator conditions for residually finite groups, Bull. London Math. Soc., 23 (1991), 239-248.

[22] J. S. Wilson, E. I. Zelmanov, Identities for Lie algebras of pro-p groups, J. Pure Appl. Algebra, 81 (1992) 103-109.

[23] E. I. Zelmanov, On the restricted Burnside problem, In: Proceedings of the International Congress of Mathematicians (1990) 395-402.

[24] E. Zelmanov, The solution of the restricted Burnside problem for groups of odd exponent, Math. USSR Izv., 36 (1991) 41-60.

[25] E. Zelmanov, The solution of the restricted Burnside problem for 2-groups, Math. Sb., 182 (1991) 568-592. 
[26] E. I. Zelmanov, Lie algebras and torsion groups with identity, J. Comb. Algebra, 1, (2017) 289-340.

(Bastos) Departamento de Matemática, Universidade de Brasília, BRASILIA-DF, 70910-900 BRAZIL

E-mail address: bastos@mat.unb.br

(Silveira) Departamento de Matemática, Universidade Federal de Goiás, Catalão-GO, 75704-020 Brazil

E-mail address: sancaodanilo@gmail.com 